\title{
How Do We End COVID-19?
}

\author{
Poornima Parameswarappa
}

CODS Journal of Dentistry (2020): 10.5005/jp-journals-10063-0068

The outbreak of coronavirus disease 2019 (COVID-19) was caused by the novel severe acute respiratory syndrome coronavirus 2 (SARS-CoV-2), which was first reported in Wuhan City, Hubei Province, China, on December 31, 2019, has spread over the world with an unsurpassable speed. The disease is so transmissible that the number of cases and deaths rose rapidly across borders, which prompted the World Health Organization (WHO) to declare the COVID-19 outbreak to be a global pandemic on March 11, 2020. As of November 26, 2020, >60,700,000 cases and 1,425,000 deaths have been reported worldwide.

The therapeutic strategies to cope with SARS-CoV-2 infection have only played a supportive role until now. Initially, the repositioning of existing drugs received much attention because it could be a short-cut compared with conventional, lengthy, and costly new drug development, but its effectiveness has not been satisfactory yet. Therefore, prevention which aimed to reduce transmission while establishing herd immunity using vaccines seems to be the key to ending the COVID-19 pandemic. ${ }^{1}$

New vaccine development typically takes $>15$ years, but SARSCoV-2 vaccines are being tested on an unprecedented fast track. As of December 2020, >140 vaccine candidates are under development with 15 of them tested in phase 3 can be grouped into five different platforms: protein subunit vaccines, viral-vectored vaccines, nucleic acid vaccines (mRNA and DNA vaccines), inactivated viruses, and live attenuated viruses. ${ }^{1}$

Not only the efficacy but also the safety of the vaccine has to be shown through large, long-term clinical trials for regulatory approval. However, the unprecedentedly accelerated development of COVID-19 vaccines has raised concerns that they could be approved with incomplete data, particularly of long-term safety in various populations. ${ }^{2}$

Public awareness and acceptance of the vaccine is another challenge. According to a public survey conducted in 19 countries ( $n=13,426$ ), only $31.9 \%$ of the respondents said they would get shots of a COVID-19 vaccine. ${ }^{3}$ Many people are still skeptical about the utility of the vaccine. This may be partly attributed to the misinformation or lack of scientifically justifiable information
Department of Pedodontics and Preventive Dentistry, College of Dental Sciences, Davangere, Karnataka, India

Corresponding Author: Poornima Parameswarappa, Department of Pedodontics and Preventive Dentistry, College of Dental Sciences, Davangere, Karnataka, India, Phone: +91 8105482841, e-mail: drpoornimas2@gmail.com

How to cite this article: Parameswarappa P. How Do We End COVID-19? CODS J Dent 2020;12(2):25.

Source of support: Nil

Conflict of interest: None

to the public. To improve the trust in COVID-19 vaccines and to increase their acceptance by the public, healthcare professionals, policymakers, and stakeholders should strive for developing messages for the public that encourage vaccination, while mitigating the harm of scientifically unjustifiable propaganda that asserts the vanity of COVID-19 vaccines. At the same time, the public should assume their own responsibility, as global citizens, for establishing a safety net against COVID-19 by actively participating in community-wide vaccination programs. The logistics of scaling up to produce the billions of doses required to immunize the world's population is an onerous task. Even if sufficient SARS-CoV-2 vaccine doses can be manufactured, worldwide delivery presents another major logistic and financial hurdle. Storage requirements will be enormous and the vaccine may need to be either frozen or refrigerated, presenting cold-chain issues.

\section{References}

1. Won $\mathrm{JH}$, Lee $\mathrm{H}$. The current status of drug repositioning and vaccine developments for the COVID-19 pandemic. Int J Mol Sci 2020;21(24):9775. DOI: 10.3390/ijms21249775.

2. Ella KM, Mohan VK. Coronavirus vaccine: light at the end of the tunnel. Indian Pediatr 2020;57(5):407-410. DOI: 10.1007/s13312-020-1812-z.

3. Lazarus JV, Ratzan SC, Palayew A, et al. A global survey of potential acceptance of a COVID-19 vaccine. Nat Med 2020;20(2):1-4. DOI: 10.1038/s41591-020-1124-9.

\footnotetext{
(c) The Author(s). 2020 Open Access This article is distributed under the terms of the Creative Commons Attribution 4.0 International License (https:// creativecommons.org/licenses/by-nc/4.0/), which permits unrestricted use, distribution, and non-commercial reproduction in any medium, provided you give appropriate credit to the original author(s) and the source, provide a link to the Creative Commons license, and indicate if changes were made. The Creative Commons Public Domain Dedication waiver (http://creativecommons.org/publicdomain/zero/1.0/) applies to the data made available in this article, unless otherwise stated.
} 